\title{
GAP FUNCTIONS AND EXISTENCE RESULTS FOR GENERALIZED VECTOR VARIATIONAL INEQUALITIES
}

\author{
YALI ZHaO, ZunQUAN XiA AND MingZHENG WANG
}

\begin{abstract}
In this paper, the gap function for a new class of generalized vector variational inequalities with point-to-set mappings (for short, GVVI) is introduced and the necessary and sufficient conditions for the GVVI are established. Furthermore, under certain pseudomonotonicity condition and hemicontinuity condition, we obtain the existence theorems for the GVVI. The results presented in this paper are new and extend corresponding results in this field.
\end{abstract}

Mathematics subject classification (2000): 49J40, 47H04, 90C29.

Key words and phrases: Generalized vector variational inequalities, point-to-set mappings, KKMmappings, gap functions, existence of solutions.

\section{REFERENCES}

[1] F. GIANNESSI, Theorems of alternative, quadratic programmes and complementarity problems, in: R. W. Cottle, F. Giannessi, J. L. Lions (Eds.), Variational Inequalities and Complementarity Problems, Wiley, Chichester, 1980, pp. 151-186.

[2] G. Y. CHEN, G. M. CHENG, Vector variational inequality and vector optimization, in: Lecture Notes in Econ. and Math. Systems, vol. 285, Springer-Verlag, Berlin, 1987, pp. 408-416.

[3] X. Q. YANG, Vector variational inequality and vector pseudo-linear optimization, J. Optim. Theory Appl. 95 (1997) 729-734

[4] G. Y. ChEN, B. D. CRAVEn, Approximate dual and approximate vector variational inequality for multiobjective optimization, J. Aust. Math. Soc. Series A 47 (1989) 418-423.

[5] E. BEHERA, L. NAYAK, On nonlinear variational-type inequality problem, Indian J. Pure Appl. Math. 30 (9) (1999) 911-923.

[6] F. Giannessi(ED.), Vector Variational Inequalities and Vector Equilibrium, Kluwer Academic Publishers, Dordrecht, Boston, London, 2000.

[7] J. LI, N. J. HuAnG, J. K. Kim, On implicit vector equilibrium problems, J. Math. Anal. Appl. 283 (2003) 437-452.

[8] X. Q. YANG, C. J. GoH, On vector variational inequality. Its application in traffic equilibria, J. Optim. Theory Appl. 95 (2)(1997) 431-443.

[9] X. Q. YANG, J. C. YAO, Gap functions and existence of solutions to set-valued vector variational inequalities, J. Optim. Theory Appl. 115 (2002) 407-417.

[10] J. LI, Z. Q. HE, Gap functions and existence of solutions to generalized vector variational inequalities, Appl. Math. Lett. 18(2005) 989-1000.

[11] R. Reemtsen, J. J. Ruckmann, Editors, Semi-Infinite Programming, Kluwer Academic Publishers, Dordrecht, Holland, 1998.

[12] S. B. NADLER JR., Multi-valued contraction mappings, Pacific J. Math. 30 (1969) 475-488.

[13] G. Y. CHEN, Existence of solutions for a vector variational inequality: an extension of HartmannStampacchia theorem, J. Optim. Theory Appl. 74 (1992) 445-456.

[14] K. FAN, A generalization of Tychonoff's fixed point theorem, Math. Ann. 142 (1961) 305-310.

[15] I. V. Konnov, J. C. YAO, On the generalized variational inequality problem, J. Math. Anal. Appl. 206 (1997) 42-58. 
[16] G. M. LeE, S. H. KuM, On implicit vector variational inequalities, J. Optim. Theory Appl. 104 (2) (2000) 409-425.

[17] K. L. Lin, D. P. YANG, J. C. YaO, Generalized vector variational inequalities, J. Optim. Theory Appl. 92 (1997) 117-125.

[18] S. J. YU, J. C. YAO, On vector variational inequalities, J. Optim. Theory Appl. 89 (1996) 749-769.

[19] A. H. SidDiQI, Q. H. ANSARI, A. KhALIQ, On vector variational inequalities, J. Optim. Theory Appl. 84 (1995) 171-180.

[20] X. Q. YANG, On vector variational inequality and its duality, Nonlinear Anal. 21 (1993) 869-877.

[21] X. Q. YANG, Generalized convex functions and vector variational inequality and its duality, J. Optim. Theory Appl. 79 (1993) 563-580. 